\title{
Systematic Review and
} Meta-Analysis to Establish the Association of Common Genetic Variations in Vitamin D Binding Protein With Chronic Obstructive Pulmonary Disease

\author{
Ritesh Khanna, Debparna Nandy and Sabyasachi Senapati * \\ Department of Human Genetics and Molecular Medicine, Central University of Punjab, Bathinda, India
}

\section{OPEN ACCESS}

Edited by:

William Scott Bush

Case Western Reserve University,

United States

Reviewed by:

Lijun Ma,

Wake Forest University, United States

Renata Ferrari,

Universidade Estadual Paulista

(UNESP), Brazil

Suzana Erico Tanni,

Universidade Estadual Paulista

(UNESP), Brazil

${ }^{*}$ Correspondence:

Sabyasachi Senapati

sabyasachi1012@gmail.com

Specialty section:

This article was submitted to Applied Genetic Epidemiology,

a section of the journal

Frontiers in Genetics

Received: 04 June 2018 Accepted: 16 April 2019

Published: 16 May 2019

Citation:

Khanna R, Nandy D and Senapati S

(2019) Systematic Review and

Meta-Analysis to Establish the

Association of Common Genetic

Variations in Vitamin D Binding Protein With Chronic Obstructive Pulmonary

Disease. Front. Genet. 10:413.

doi: 10.3389/fgene.2019.00413
Background: Vitamin-D binding protein (DBP) also known as GC protein, is a major determinant for vitamin- D metabolism and transport. GC1F, GC1S, and GC2 are the three allelic variants (denoted as rs4588 and rs7041) of GC, and known to be associated with chronic obstructive pulmonary disease (COPD). However, contradictory reports and population specific risk attributed by these alleles warranted detailed genetic epidemiology study to establish the association between GC variants and COPD. In this study we performed a meta-analysis and investigated the genetic architecture of GC locus to establish the association and uncover the plausible reason for allelic heterogeneity.

Methods: Published cross-sectional case control studies were screened and meta-analysis was performed between GC variants and COPD outcome. RevMan-v5.3 software was used to perform random and/or fixed models to calculate pooled odds ratio (Meta-OR). Linkage disequilibrium (LD) and haplotypes at GC locus were evaluated using 1000 Genomes genotype data. In silico functional implications of rs4588 and rs7041 was tested using publicly available tools.

Results: GC1F allele and GC1F/1F genotype were found to confer COPD risk in overall meta-analysis. GC1S/1S was found to confer risk only among Europeans. In silico investigation of rs4588 and rs7041 identified strong eQTL effects and potential role in regulation of GC expression. Large differences in allele frequencies, linkage disequilibrium (LD) and haplotypes were identified at GC locus across different populations (Japanese, African, Europeans, and Indians), which may explain the variable association of different $\mathrm{GC}$ alleles in different populations.

Conclusion: GC1F and GC1F/1F impose significant genetic risk for COPD, among Asians. Considerable differences in allele frequencies and LD structure in GC locus may impose population specific risk.

Keywords: vitamin D-binding protein, COPD, meta-analysis, linkage disequilibrium, genetic polymorphisms, allelic heterogeneity 


\section{INTRODUCTION}

Chronic obstructive pulmonary disease (COPD) is a complex disease affecting the lung function. Genetically susceptible individuals develop the COPD while they get exposed to environmental triggers, such as noxious gases or suspended particles. Decreased level of vitamin-D in serum is associated with COPD among individuals with a history of smoking (Janssens et al., 2010). Besides environmental and genetic factors, metabolic factors are also critical and do cross talk with each other for the pathogenesis of COPD (Rabe et al., 2007).

Vitamin-D binding protein (DBP), also known as groupspecific component (GC), belongs to a gene cluster family which is expressed in liver and other tissues (Chishimba et al., 2010). As the name suggests, it is known for its binding to circulating vitamin- $\mathrm{D}_{3}$ and its transportation from liver to other tissues during its metabolism (Daiger et al., 1975). GC is a highly polymorphic gene and three of its allelic variants, namely GC1F, GC1S, and GC2, have been studied extensively for their association with vitamin-D deficiency (VDD) and other diseases including COPD (Chishimba et al., 2010; Wood et al., 2011). These variants correspond to different allelic arrangements of rs7041 and rs4588 (Table 1). These GC protein variants are reported to have a different affinity to bind to vitamin- $\mathrm{D}_{3}$ i.e., $25(\mathrm{OH}) \mathrm{D}_{3}$, and thus affect its serum concentration (Arnaud and Constans, 1993; Janssens et al., 2010). Circulating level of vitamin- $\mathrm{D}_{3}$ is regulated by the synthesis and enzymatic degradation of $25(\mathrm{OH}) \mathrm{D}_{3}$ by catabolizing enzymes. More than $90 \%$ of the circulating $25(\mathrm{OH}) \mathrm{D}_{3}$ present in tightly bound $\left(\mathrm{K}_{\mathrm{d}} \sim 10^{-9} \mathrm{M}\right)$ form with GC proteins (Arnaud and Constans, 1993). Therefore, different GC isoforms influence the serum concentration/bioavailability of $25(\mathrm{OH}) \mathrm{D}_{3}$.

A study performed on north Indian cohort has shown homozygous GC1F variant to confer risk, and the disease severity is observed in a variant specific dose dependent manner, where the geometric mean of serum $25(\mathrm{OH}) \mathrm{D}_{3}$ was observed in the ascending order of $G C$ genotypes $1 \mathrm{~F} / 1 \mathrm{~F}<1 \mathrm{~S} / 1 \mathrm{~F}<1 \mathrm{~S} / 1 \mathrm{~S}<1 \mathrm{~S} / 2<2 / 2$ among $\mathrm{COPD}$ patients (Maheswari et al., 2014). Recent reports also indicate the protective role of GC2 variant among healthy individuals. Similar reports were published by the studies done among Caucasian and north Indian cohorts (Schellenberg et al., 1998; Berg et al., 2013; Maheswari et al., 2014; Chen et al., 2015). Azzawi et al. confirmed similar study outcomes in

TABLE 1 | Allelic arrangements correspond to three different GC variants implicated in COPD.

\begin{tabular}{lll}
\hline GC variants & $\begin{array}{l}\text { Allele of rs4588 (amino } \\
\text { acid) }\end{array}$ & $\begin{array}{l}\text { Allele of rs7041(amino } \\
\text { acid) }\end{array}$ \\
\hline GC-1F & C & T \\
& $($ Thr436) & (Asp432) \\
GC-1S & C & A/G \\
& $($ Thr436) & (Glu432) \\
GC-2 & A & $T$ \\
& $($ Lys436) & (Asp432)
\end{tabular}

an Egyptian cohort where GC1F and GC1F/1S variants were found to be associated with low serum vitamin- $\mathrm{D}_{3}$ concentration (Al-Azzawi et al., 2017). While studies done among Korean population have shown an association of GC2 variants with COPD progression, where GC2 and GC1F/1S variants were shown to be associated with higher emphysema index, irrespective of VDD. These studies also identified an association of $G C 2$ and $G C 1 F / 1 S$ variants with lower and higher serum concentration of vitamin- $\mathrm{D}_{3}$, respectively. GC2 showed significant association with VDD (Jung et al., 2014; Park et al., 2016).

GC protein (or DBP) is also involved in the inflammation by getting converted into MAF (Macrophage Activating Factor) in the presence of enzymes secreted by leucocytes. It has been found that the conversion of GC into GC-MAF is a deglycosylation process. Absence of glycosylated Lys residue at 420 in GC2 variants makes it an inappropriate reactant for the deglycosylation process, which makes them protective for COPD (Maheswari et al., 2014). Vitamin- $\mathrm{D}_{3}$ is known to inhibit the expression of MMPs (Matrix Metalloproteinases), which are responsible for the emphysema degradation of lung alveoli. Thus, optimal serum concentration of Vitamin- $\mathrm{D}_{3}$ is very critical among emphysema patients and a trial for such serum Vitamin $\mathrm{D}_{3}$ intervention among a large participant group can further elucidate its role in COPD progression (Berg et al., 2013). Serum Vitamin- $\mathrm{D}_{3}$ is also found to have seasonal and geographical variations, which depend on the amount of sunlight reaching the skin (Jung et al., 2014; AlAzzawi et al., 2017). ECLIPSE Cohort study did not find an association between serum DBP and emphysema or lung function, although a negative correlation was found among DBP and serum $25(\mathrm{OH}) \mathrm{D}_{3}$ level (Berg et al., 2013). While another study in an alphal-antitrypsin deficient Caucasian population showed the association of serum DBP with COPD conditions (Wood et al., 2011). A recent report indicated a strong relationship between serum $25(\mathrm{OH}) \mathrm{D}_{3}$ and pulmonary function (FEV1 and FVC) in a well-defined COPD cohort (Janssens et al., 2010).

It is evident that GC is a major determinant for several health parameters including those associated with COPD. However, contradictory findings of association of different alleles with COPD and non-replication across different populations warranted further meta-analysis and detailed population genetics studies. In the present study, we anticipated to explain the association of known GC alleles with COPD and investigate the genetic and functional aspects of $G C$ alleles. Locus architecture of different populations was also investigated to explain the non-replication/differential replication of $G C$ alleles in different populations. We hypothesized that genetics architecture at $G C$ locus leads to population specific allelic variation in $G C$ and its association with COPD. The study was performed with the following specific objectives: (i) perform meta-analysis to establish association of commonly studied GC alleles with COPD, and (ii) investigate the genetic heterogeneity at a functionally relevant GC locus, that explain variability in GC protein and COPD. 


\section{MATERIALS AND METHODS}

\section{Literature Retrieval}

Our objective was to identify research articles where genetic association of $G C$ has been tested with COPD. We restricted our study to three major genetic polymorphisms of $G C$, namely GC1F, GC1S, and GC2 alleles. Literature was searched online in the National Center for Biotechnology Information (NCBIPubMed), Google Scholar and Medline. The major search language for the literature was English, papers in other languages were translated for further review. To obtain the best quality outcome, we include only peer reviewed scientific literature. Literature were searched until May 2018. The keywords used for the search for literature were as follows: Vitamin D binding protein and chronic obstructive pulmonary disease, DBP and COPD, GC alleles and COPD, COPD association GC. Cross references were also reviewed and references from the retrieved articles were also checked manually so as to find any relevant articles.

\section{Inclusion and Exclusion Criteria}

Only case-control studies were included for this meta-analysis. Only those studies were included where different alleles $(1 \mathrm{~F}, 1 \mathrm{~S}$, and 2 ) and genotypes (1F/1F, 1S/1S, 2/2, 1F/1S, 1F/2, 1S/2) of GC were studied for their association with COPD. Included studies clearly mentioned either the actual numbers, or the percentage of cases and controls with different genotypes and alleles of GC. Included studies have both smokers and non-smokers among both cases and controls.

\section{Data Extraction}

Data was extracted from eligible articles by two investigators independently and differences and controversies were resolved by group discussions. We first validated the study types and then extracted author names, year of publication, details of genotypes/alleles and their frequencies in COPD patients and controls.

\section{Statistical Analysis}

Results of association of three distinct alleles have been included in this study. These alleles were GC1F, GC1S and GC2, represented in NCBI dbSNP as rs4588 and rs7041, respectively (Table 1). Therefore, a total of six different genotypic combinations were studied, such as, GC1F/1F, GC1F/1S, $G C 1 S / 1 S, G C 1 F / 2, G C 1 S / 2$, and $G C 2 / 2$. Independently these genotypes and three allelic associations were evaluated by metaanalysis. In each analysis, the experimental allele or genotypes were tested against the total allele or genotype counts. Metaanalysis was performed using Review Manager (RevMan-v5.3) Copenhagen: The Nordic Cochrane Center, The Cochrane Collaboration, 2014. Additive genetic model with 95\% confidence interval (CI) was used in each of these independent analyses. Heterogeneity between studies was calculated by the $I^{2}$ and $\mathrm{chi}^{2}$ test, where $I^{2}>50 \%$ and $\mathrm{chi}^{2} p<0.05$ was considered as significant heterogeneity. Meta-analysis of odds ratios were performed using a random effect model where significant heterogeneity was observed, otherwise a fixed effect model was used. Overall effect size (Meta-OR) was calculated by Z-test with
5\% alpha level. A sensitivity analysis was performed to access whether meta-analysis results were substantially influenced by the presence of any study. This was done by systematically excluding one study at a time and recalculating the significance ( $p$-value of the $\chi^{2}$ and $Z$-test) of the results. The funnel plot was used to analyze the publication bias. Subgroup analysis between Asian and Caucasian studies was also performed to identify any significant differences due to individual group stratification.

\section{Linkage Disequilibrium, Haplotypes, and Comparative Allele Frequency}

Genetic architecture of GC locus was evaluated to explain population specific effects (if any) of $G C$ alleles on its association with different human traits/diseases. To analyze the linkage disequilibrium, LD plots and haplotypes were reconstructed using Haploview (Barrett et al., 2004). LD calculations and manipulation of genotype files were done using Plink 1.07 (Purcell et al., 2007). 1000 Genomes genotype information for four major populations, such as CEU (Utah residents with northern and western European ancestry), GIH (Gujarati Indians in Houston, USA), YRI (Yoruba in Ibadan, Nigeria), and JPT (Japanese in Tokyo), were evaluated for LD analysis. Raw genotype data for these populations were obtained from 1,000 Genomes ftp through Ensembl. Genotype data were obtained for a $50 \mathrm{~kb}$ window on both the sides around rs7041 i.e., chr4:71702617-71802617 (GRCh38.p12). Comparative allele frequencies for GC1F, GC1S, and GC2 corresponding to rs 4588 and rs7041 were evaluated from Ensembl (https://asia.ensembl. org/index.html), HaploReg (http://archive.broadinstitute.org/ mammals/haploreg/haploreg.php).

\section{In silico Functional Implication Assessment} Functional implications of rs4588 and rs7041 were analyzed using open source browsers. RegulomeDB (http://www. regulomedb.org/index) was used to analyze the regulatory function and GTEx portal (https://gtexportal.org/home/) was used to analyze single tissue or gene eQTL.

\section{RESULTS}

\section{Characteristics of Eligible Studies}

A total of 71 studies were identified initially after online literature search. After screening and proper reviewing for the eligible papers 48 papers were excluded. There were two duplicate studies, six studies were for asthma, and 11 were for diseases other than asthma and COPD, such as osteomalacia, type II diabetes, adenocarcinoma, pulmonary tuberculosis and other non-relevant diseases. There was a non-human study done on mice, which was also excluded from the meta-analysis. Metaanalysis $(n=5)$, which was done previously on COPD and $G C$, was also excluded but was used to identify cross references. Fourteen studies were excluded because they were either cohort studies or random clinical trials done on supplementation of vitamin- $\mathrm{D}_{3}$. Eleven studies were found irrelevant, either due to less information for cases or control subjects, and one study was in other language and was excluded from the meta-analysis. A further seven studies were excluded as adequate/complete 
genotype and study participant information were not given. After this screening based on our inclusion/exclusion criteria, a total of 14 studies were found eligible for meta-analysis (Kueppers et al., 1977; Home et al., 1990; Ishii et al., 2001; Ito et al., 2004; Laufs et al., 2004; Lu et al., 2004; Korytina et al., 2006; Huang et al., 2007; Janssens et al., 2010; Shen et al., 2010; Jung et al., 2014; Li et al., 2014; Maheswari et al., 2014; Al-Azzawi et al., 2017) (Figure 1).

\section{Genotypic and Allelic Association}

A total of 14 studies were included in this meta-analysis where genotypes for different above-mentioned $G C$ alleles, in both COPD patients and healthy controls, were reported. Out of these 14 studies, nine studies were performed on different Asian populations and five were on European populations. Details of the study participants and haplotypes or allele frequencies are given in the Supplementary Table 1. Random effect model was performed to find out the pooled effect size for $G C 1 \mathrm{~F} / 1 \mathrm{~F}$, $G C 1 \mathrm{~F} / \mathrm{S}, G C 1 \mathrm{~F} / 2$, and $G C 2 / 2$ genotypes, and GC1F, GC1S and $G C 2$ alleles in COPD. For remaining analyses, fixed effect model was used due to insignificant study heterogeneity $\left(\mathrm{chi}^{2} p>0.05\right.$ and $I^{2}<50 \%$ ) (Figure 2 and Supplementary Figure 1). Metaanalysis was performed separately for reports on Asians and Europeans to identify significant differences in effect size, if any.

\section{Allelic Association}

GC1F allele has been found significantly predisposing for COPD outcome in combined analysis (Meta-OR $=1.29 ; 95 \%$ $\mathrm{CI}=1.09-1.55 ; \mathrm{Z}$ p-val = 0.004). Independently, GC1F allele has been found strongly associated among Asians $\left(\mathrm{OR}_{\text {Asia }}=1.45\right.$; $95 \% \mathrm{CI}=1.24-1.68 ; \mathrm{Z}$ p-val $<0.00001)$, but not among Europeans $\left(\mathrm{OR}_{\text {Europe }}=1.02 ; 95 \% \mathrm{CI}=0.73-1.42 ; \mathrm{Z} \mathrm{p}\right.$ val $=0.92)($ Figures 2A,B). Both GC1S and GC2 alleles were not found significant in conferring risk or protection with COPD outcome (Supplementary Figure 1). However, considering the trend of association, both these alleles were found protective in combined analyses.

\section{Genotypic Association}

Homozygous GC1F/1F was found significantly predisposing genotype with COPD outcome (Meta-OR $=1.61$; $95 \% \mathrm{CI}=1.18-$ $2.20 ; \mathrm{Z}$ p-val $=0.002)$. Independent analysis found significant association of this genotype among Asians $\left(\mathrm{OR}_{\mathrm{Asia}}=1.93 ; 95 \%\right.$ $\mathrm{CI}=1.38-2.70 ; \mathrm{Z}$-val $=0.0001)$, but it remains insignificant among Europeans $\left(\mathrm{OR}_{\text {Europe }}=1.11\right.$ with $95 \% \mathrm{CI}=0.64-1.95$; $\mathrm{Z}$ p-val $=0.71)$. Significant predisposition was observed for GC1S/1S genotype among Europeans (OR Europe $=1.29 ; 95 \%$ $\mathrm{CI}=1.00-1.68 ; \mathrm{Z}$-val $=0.05)$, however it remains insignificant among Asians (Figure 2). Further, no significant associations were observed for any of the alleles or genotypes, either in combined or independent analyses in Asians and Europeans (Supplementary Figure 1).

\section{Sensitivity Analysis and Publication Bias}

Sensitivity analysis was performed for each study. No significant deviation in heterogeneity and study significance ( $p$-value of the $\chi^{2}$ and $Z$-test) was observed. Subgroup analyses did not identify any significant $(\mathrm{p}<0.05)$ subgroup stratification (Figure 2 and
Supplementary Figures 1A-G). Further, manual investigation of funnel plots did not identify any publication bias, where shapes of the funnel plots were symmetrical (Supplementary Figure 2).

\section{Linkage Disequilibrium}

Comparative LD analysis of GC locus showed substantial differences in the background LD structure between four reference populations. Comparatively similar LD structure was observed in CEU and GIH, however structure is further broken in JPT and YRI. Both the variations, rs4588 and rs7041, do not constitute any likely haplo-blocks in JPT and YRI (Supplementary Figure 3). Haplotypes for GC1F, GC1S, and $G C 2$ were found to be present with relatively equal frequency among CEU $(0.19,0.57$, and 0.24$)$ and $\operatorname{GIH}(0.21,0.46$, and 0.32 ), however, these haplotypes were not found in JPT and YRI. Furthermore, moderate yet similar LD was observed between these two markers in CEU $\left(r^{2}=0.42\right.$; D' $\left.=1\right)$ and GIH $\left(r^{2}=0.41\right.$; $\left.\mathrm{D}^{\prime}=1\right)$, however, LD is completely broken in JPT $\left(r^{2}=0.10\right.$; $\left.\mathrm{D}^{\prime}=1\right)$ and YRI $\left(r^{2}=0.00 ; \mathrm{D}^{\prime}=0.53\right)$. Notable haplotypic variations were observed across the genomic region, whereas in JPT and YRI, these two variations are not in tight linkage with neighboring markers (Supplementary Figure 3). Allele frequencies of rs4588 and rs7041 and LD between them were seen to be very heterogeneous across 26 different populations, as documented in 1000 Genomes Project. Absolutely no LD $\left(r^{2}=0\right)$ was observed among different African populations, whereas the highest degree of LD was observed among Europeans and South Asian populations followed by Americans (Supplementary Table 2).

\section{In silico Functional Implications}

GC (ENSG00000145321) expresses in the liver despite very negligible expression in the pancreas and stomach. For two missense SNPs, rs4588, and rs7041, no evidence was observed for significant eQTL on $G C$ in liver tissue, however, significant eQTL was observed in subcutaneous adipose $(p=6.55 \mathrm{E}-6)$, sun exposed skin $(p=1.67 \mathrm{E}-6)$, and stomach $(p=5.46 \mathrm{E}-$ 9) tissues. SNP rs4588 was identified to alter motif-binding sites of transcription factors SP1 and SP3; and transcription factor binding element (KLF16). rs4588 and rs7041 were both identified: (a) to localize in DNase hypersensitivity regions in a common set of cell types and tissues, and (b) potentially alter histone modification in liver (strongly) and skin (quiescent/low) tissue.

\section{DISCUSSION}

In this systematic review, we performed meta-analysis and evaluated linkage disequilibrium at GC locus, in order to investigate the association of common $G C$ polymorphisms with COPD. This meta-analysis established that GC1F allele and GC1F/1F genotype confers risk of COPD. However, association is majorly restricted to Asians and not in Europeans. On the contrary, GC1S/1S genotype was observed to confer risk to Europeans only (with borderline significance). At least one copy of $G C 2$ has been found to confer protection from COPD among both Asians and Europeans. Previous meta-analysis studies 


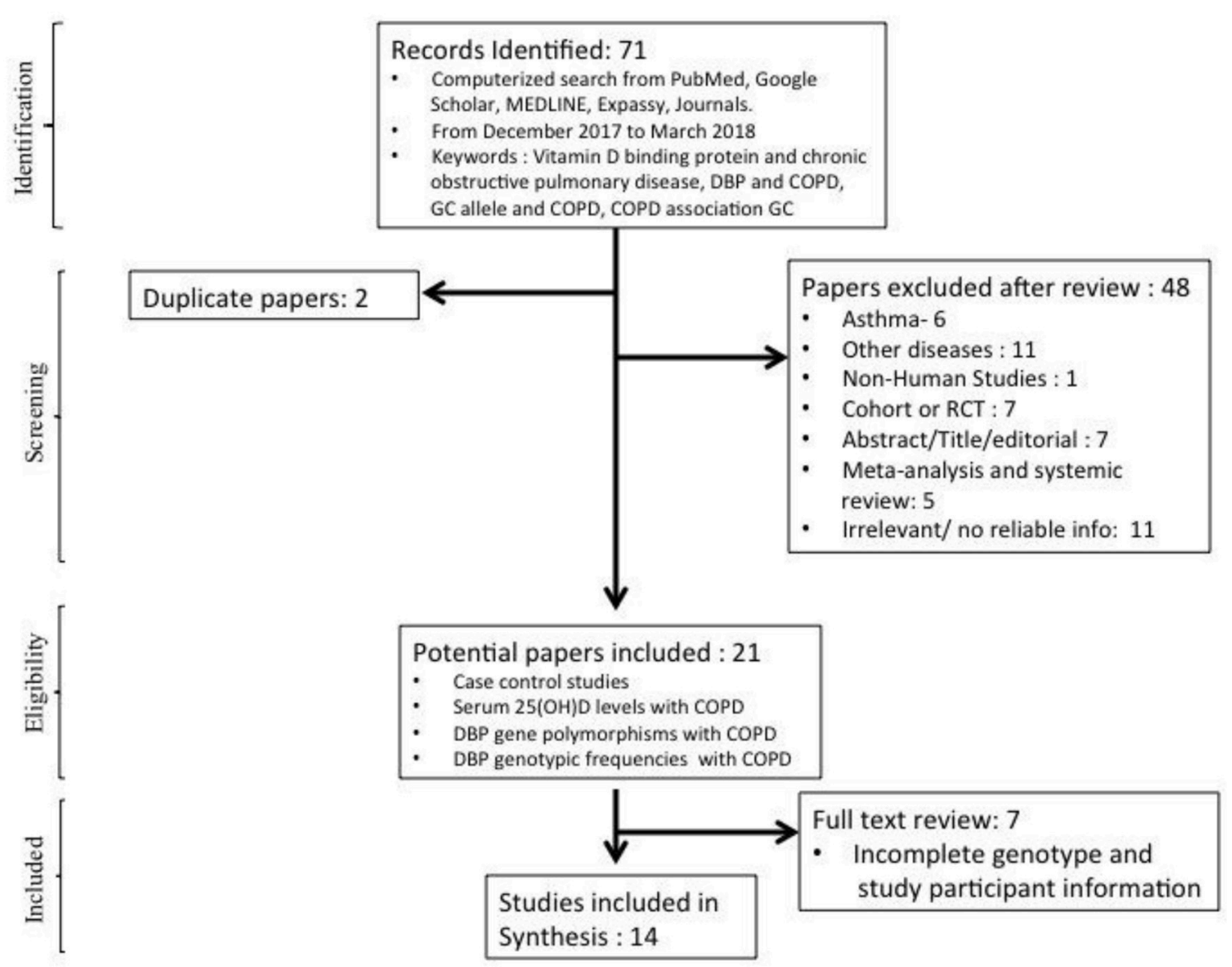

FIGURE 1 | Step-wise flow chart showing identification and screening of studies included in the meta-analysis.

and independent reports have shown different results from Europeans and Asians, which could be due to differences in allelic segregation and haplotypic heterogeneity at a population level (Chen et al., 2015; Horita et al., 2015; Wang et al., 2015; Xiao et al., 2015; Xie et al., 2015). Large differences in LD structure and haplotypes were observed in different ethnic populations, such as CEU GIH, JTP, and YRI. Although no reports are available (on association of $G C$ variants and COPD) from African countries, we have included their representative genotypes for comparative genetic studies (Supplementary Figure 3). Notable differences in allele frequencies and LD between rs4588 and rs7041, among different populations, suggest significant population specific genetic contribution in $G C$ variants (Supplementary Table 2). These major differences in LD between these two critical variants resulted into different haplotype frequencies and an absence of any quantifiable haplotypes in JPT and YRI. This indicates that perhaps different haplotypes are associated with different ethnic populations, which requires further large-scale genetic studies to uncover the novel alleles or haplotypes, if any. The overall trend shows relative similarity between CEU and GIH and distinct differences were observed in YRI and JPT. Different allelic arrangements of GC result into different GC variants, which vary in their isoelectric points and binding efficiency to vitamin $D_{3}$ (Braun et al., 1992; Arnaud and Constans, 1993; Speeckaert et al., 2006). Furthermore, in different populations, rs4588 and rs7041 may tag different sets of regulatory and structural SNPs (in haplotypes) across GC, and thus could play critical role in regulating expression and function of the GC protein.

Although VDD is found to be associated with COPD (Jolliffe et al., 2018), the underlying causes for such mechanisms remain unanswered. Recent GWAS studies on COPD were unable to identify GC or vitamin D receptor (VDR) as a significantly associated gene (Wain et al., 2017). However, genetic polymorphisms from these genes are found to be associated with VDD (Yousefzadeh et al., 2014; Zaki et al., 2017). In most of the studies, low level of serum Vitamin- $\mathrm{D}_{3}$ is reported to be associated with the severity of COPD condition. Particularly, rs4588 has been shown to influence GC binding to Vitamin$\mathrm{D}_{3}$ (Nimitphong et al., 2013). It can be argued that, along with sufficient vitamin- $\mathrm{D}_{3}$ intake/supplementation, a functionally more potent form of GC is necessary to maintain optimal serum bioavailability of vitamin- $\mathrm{D}_{3}$. Therefore, inter individual differences in the GC protein may act as a predisposing factor for COPD. Further genetic epidemiological studies are warranted to identify novel risk alleles from $G C$ that are associated with $G C$ 


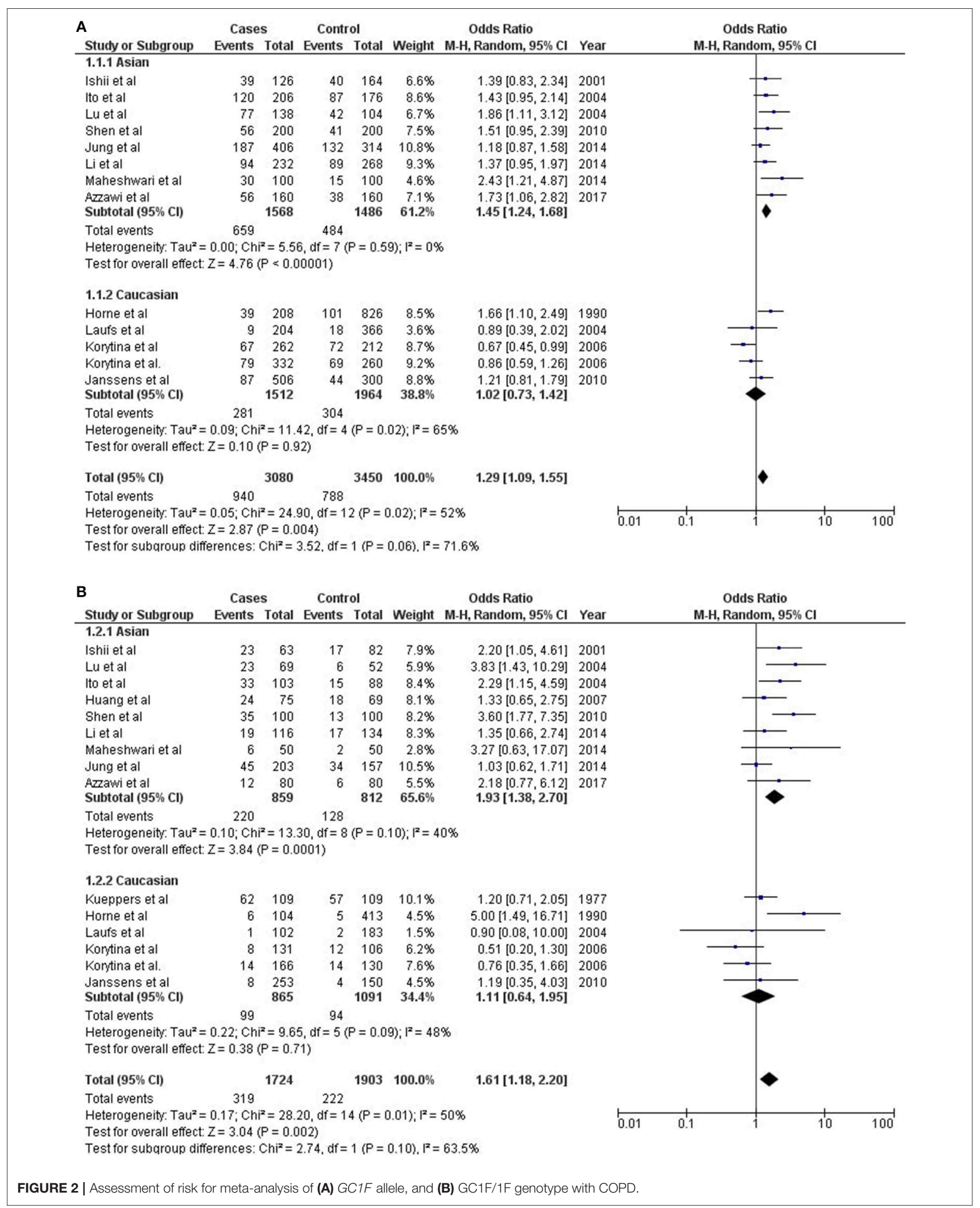


function, and thus implication in COPD. However, the presence of differential LD structure of GC locus needs to be considered as a major confounding factor.

\section{AUTHOR CONTRIBUTIONS}

SS conceptualized and designed the study. RK and DN performed literature screening and meta-analysis. SS performed in silico genetic study. RK, DN, and SS contributed in writing the manuscript and interpreted the results. All the authors reviewed the manuscript and finalized for submission.

\section{REFERENCES}

Al-Azzawi, M. A., Ghoneim, A. H., and Elmadbouh, I. (2017). Evaluation of vitamin D, vitamin D binding protein gene polymorphism with oxidantantioxidant profiles in chronic obstructive pulmonary disease. J. Med. Biochem. 36, 331-340. doi: 10.1515/jomb-2017-0012

Arnaud, J., and Constans, J. (1993). Affinity differences for vitamin D metabolites associated with the genetic isoforms of the human serum carrier protein (DBP). Hum. Genet. 92, 183-188. doi: 10.1007/BF00219689

Barrett, J. C., Fry, B., Maller, J., and Daly, M. J. (2004). Haploview: analysis and visualization of LD and haplotype maps. Bioinformatics 21, 263-265 doi: 10.1093/bioinformatics/bth457

Berg, I., Hanson, C., Sayles, H., Romberger, D., Nelson, A., Meza, J., et al. (2013). Vitamin D binding protein, lung function and structure in COPD. Respir. Med. 107, 1578-1588. doi: 10.1016/j.rmed.2013.05.010

Braun, A., Bichlmaier, R., and Cleve, H. (1992). Molecular analysis of the gene for the human vitamin-D-binding protein (group-specific component): allelic differences of the common genetic GC types. Hum. Genet. 89, 401-406. doi: 10.1007/BF00194311

Chen, H., Zhang, L., He, Z., Zhong, X., Zhang, J., Li, M., et al. (2015). Vitamin D binding protein gene polymorphisms and chronic obstructive pulmonary disease: a meta-analysis. J. Thoracic Dis. 7:1423-1440. doi: 10.3978/j.issn.2072-1439.2015.08.16.

Chishimba, L., Thickett, D. R., Stockley, R. A., and Wood, A. M. (2010). The vitamin $\mathrm{D}$ axis in the lung: a key role for vitamin D-binding protein. Thorax 65, 456-462. doi: 10.1136/thx.2009.128793

Daiger, S. P., Schanfield, M. S., and Cavalli-Sforza, L. L. (1975). Group-specific component (GC) proteins bind vitamin D and 25-hydroxyvitamin D. Proc. Nat. Acad. Sci. U.S.A. 72, 2076-2080.

Home, S. L., Cockcroft, D. W., and Dosman, J. A. (1990). Possible protective effect against chronic obstructive airways disease by the GC 2 allele. Hum. Hered. 40, 173-176. doi: 10.1159/000153926

Horita, N., Miyazawa, N., Tomaru, K., Inoue, M., Ishigatsubo, Y., and Kaneko, T. (2015). Vitamin D binding protein genotype variants and risk of chronic obstructive pulmonary disease: a meta-analysis. Respirology 20, 219-225. doi: $10.1111 /$ resp. 12448

Huang, P., Ma, Y., Du, X., Chen, J., Song, B., Hong, Y., et al. (2007). The vitamin Dbinding protein gene polymorphism in chronic obstructive pulmonary disease patients. Zhonghua Jie He He Hu Xi ZaZhi. 30, 780-781.

Ishii, T., Keicho, N., Teramoto, S., Azuma, A., Kudoh, S., Fukuchi, Y., et al. (2001). Association of Gc-globulin variation with susceptibility to COPD and diffuse panbronchiolitis. Eur. Respir. J. 18, 753-757. doi: 10.1183/09031936.01.00094401

Ito, I., Nagai, S., Hoshino, Y., Muro, S., Hirai, T., Tsukino, M., et al. (2004). Risk and severity of COPD is associated with the group-specific component of serum globulin 1F allele. Chest 125, 63-70. doi: 10.1378/chest.125.1.63

Janssens, W., Bouillon, R., Claes, B., Carremans, C., Lehouck, A., Buysschaert, I., et al. (2010). Vitamin D deficiency is highly prevalent in COPD and correlates with variants in the vitamin D-binding gene. Thorax 65, 215-220. doi: 10.1136/thx.2009.120659

\section{FUNDING}

We acknowledge financial supports from DST-SERB (\#ECR/2016/001660), UGC-BSR grant (30-4/2014-BSR), and research grant from Central University of Punjab (GP.25).

\section{SUPPLEMENTARY MATERIAL}

The Supplementary Material for this article can be found online at: https://www.frontiersin.org/articles/10.3389/fgene. 2019.00413/full\#supplementary-material

Jolliffe, D. A., James, W. Y., Hooper, R. L., Barnes, N. C., Greiller, C. L., Islam, K., et al. (2018). Prevalence, determinants and clinical correlates of vitamin D deficiency in patients with Chronic Obstructive Pulmonary Disease in London, U. K. J. Steroid Biochem. Mol. Biol. 175, 138-145. doi: 10.1016/j.jsbmb.2017.01.019

Jung, J. Y., Choi, D. P., Won, S., Lee, Y., Shin, J. H., Kim, Y. S., et al. (2014). Relationship of vitamin D binding protein polymorphisms and lung function in Korean chronic obstructive pulmonary disease. Yonsei Med. J. 55, 1318-1325. doi: 10.3349/ymj.2014.55.5.1318

Korytina, G. F., Akhmadishina, L. Z., Ianbaeva, D. G., and Viktorova, T. V. (2006). Genotypes of vitamin-D-binding protein (DBP) in patients with chronic obstructive pulmonary disease and healthy population of Republic Bashkortostan. Mol. Biol. 40, 231-238. doi: 10.1134/S002689330602004X

Kueppers, F., Miller, R. D., Gordon, H., Hepper, N. G., and Offord, K. (1977). Familial prevalence of chronic obstructive pulmonary disease in a matched pair study. Am. J. Med. 63, 336-342. doi: 10.1016/0002-9343(77)90270-4

Laufs, J., Andrason, H., Sigvaldason, A., Halapi, E., Thorsteinsson, L., Jónasson, K., et al. (2004). Association of vitamin D binding protein variants with chronic mucus hypersecretion in Iceland. Am. J. Pharmacogenom. 4, 63-68. doi: 10.2165/00129785-200404010-00007

Li, X., Liu, X., Xu, Y., Xiong, W., Zhao, J., Ni, W., et al. (2014). The correlation of vitamin $\mathrm{D}$ level and vitamin $\mathrm{D}$-binding protein gene polymorphism in chronic obstructive pulmonary disease. Zhonghuaneikezazhi 53, 303-307.

Lu, M., Yang, B., and Cai, Y. Y. (2004). The relationship between vitamin $\mathrm{D}$ binding protein gene polymorphism and chronic obstructive pulmonary disease. ZhonghuaNeiKeZaZhi 43, 117-120.

Maheswari, K., Choudhary, M., Javid, S. (2014). Association of Vitamin D Binding protein gene polymorphism with serum 25-hydroxy Vitamin D levels in COPD. Online Int. Interdiscipl. Res. J. 4, 46-55.

Nimitphong, H., Saetung, S., Chanprasertyotin, S., Chailurkit, L. O., and Ongphiphadhanakul, B. (2013). Changes in circulating 25-hydroxyvitamin $\mathrm{D}$ according to vitamin $\mathrm{D}$ binding protein genotypes after vitamin D3or vitamin D2 supplementation. Nutr. J. 4:1239. doi: 10.1186/1475-289112-39

Park, Y., Kim, Y. S., Kang, Y. A., Shin, J. H., Oh, Y. M., Seo, J. B., et al. (2016). Relationship between vitamin D-binding protein polymorphisms and blood vitamin D level in Korean patients with COPD. Int. J. Chron. Obstruct. Pulmon Dis. 11, 731-738. doi: 10.2147/COPD.S96985

Purcell, S., Neale, B., Todd-Brown, K., Thomas, L., Ferreira, M. A., Bender, D., et al. (2007). PLINK: a tool set for whole-genome association and populationbased linkage analyses. Am. J. Hum. Genet. 81, 559-575. doi: 10.1086/ 519795

Rabe, K. F., Hurd, S., Anzueto, A., Barnes, P. J., Buist, S. A., Calverley, P., et al. (2007). Global strategy for the diagnosis, management, and prevention of chronic obstructive pulmonary disease: GOLD executive summary. Am J RespirCrit Care Med. 176, 532-555. doi: 10.1164/rccm.200703-456SO

Schellenberg, D., Paré, P. D., and Weir, T. D., Spinelli, J. J., Walker, B. A., and Sandford, A. J. (1998). Vitamin D binding protein variants and the risk of COPD. Am. J. Respir. Crit. Care Med. 157, 957-961. doi: 10.1164/ajrccm.157.3.9706106 
Shen, L. H., Zhang, X. M., Su, D. J., Yao, S. P., Yu, B. Q., Wang, H. W., et al. (2010). Association of vitamin D binding protein variants with susceptibility to chronic obstructive pulmonary disease. J. Int. Med. Res. 38, 1093-1098. doi: $10.1177 / 147323001003800337$

Speeckaert, M., Huang, G., Delanghe, J. R., and Taes, Y. E. (2006). Biological and clinical aspects of the vitamin D binding protein)Gc-globulin) and its polymorphism. Clin. Chim. Acta. 372, 33-42. doi: 10.1016/j.cca.2006.03.011

Wain, L. V., Shrine, N., Artigas, M. S., Erzurumluoglu, A. M., Noyvert, B., BossiniCastillo, L., et al. (2017). Genome-wide association analyses for lung function and chronic obstructive pulmonary disease identify new loci and potential druggable targets. Nat. Genet. 49, 416. doi: 10.1038/ng.3787

Wang, Y. L., Kong, H., Xie, W. P., and Wang, H. (2015). Association of vitamin Dbinding protein variants with chronic obstructive pulmonary disease: a metaanalysis. Genet. Mol. Res. 14, 10774-10785. doi: 10.4238/2015.September.9.16

Wood, A. M., Bassford, C., Webster, D., Newby, P., Rajesh, P., Stockley, R. A., et al. (2011). Vitamin D-binding protein contributes to COPD by activation of alveolar macrophages. Thorax 66, 205-210. doi: 10.1136/thx.2010.140921

Xiao, M., Wang, T., Zhu, T., and Wen, F. (2015). Dual role of vitamin D-binding protein $1 \mathrm{~F}$ allele in chronic obstructive pulmonary disease susceptibility: a meta-analysis. Genet. Mol. Res. 14, 3534-3540. doi: 10.4238/2015.April.17.1

Xie, X., Zhang, Y., Ke, R., Wang, G., Wang, S., Hussain, T., et al. (2015). Vitamin D-binding protein gene polymorphisms and chronic obstructive pulmonary disease susceptibility: a meta-analysis. Biomed. Rep. 3, 183-188. doi: 10.3892/br.2014.392

Yousefzadeh, P., Shapses, S. A., and Wang, X. (2014). Vitamin D binding protein impact on 25-hydroxyvitamin $\mathrm{D}$ levels under different physiologic and pathologic conditions. Int. J. Endocrinol. 2014:981581. doi: 10.1155/2014/981581

Zaki, M., Kamal, S., Basha, W. A., Youness, E., Ezzat, W., El-Bassyouni, H., et al. (2017). Association of vitamin D receptor gene polymorphism (VDR) with vitamin D deficiency, metabolic and inflammatory markers in Egyptian obese women. Genes Dis. 4, 176-182. doi: 10.1016/j.gendis.2017.07.002

Conflict of Interest Statement: The authors declare that the research was conducted in the absence of any commercial or financial relationships that could be construed as a potential conflict of interest.

Copyright (๑) 2019 Khanna, Nandy and Senapati. This is an open-access article distributed under the terms of the Creative Commons Attribution License (CC BY). The use, distribution or reproduction in other forums is permitted, provided the original author(s) and the copyright owner(s) are credited and that the original publication in this journal is cited, in accordance with accepted academic practice. No use, distribution or reproduction is permitted which does not comply with these terms. 